\title{
A decomposition formula for the wall heat flux of a compressible boundary layer
}

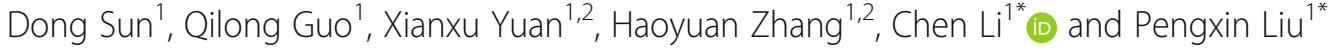

\author{
* Correspondence: lichen@skla. \\ cardc.cn; liupengxin@cardc.cn \\ ${ }^{1}$ State Key Laboratory of \\ Aerodynamics, Mianyang 621000, \\ Sichuan, China \\ Full list of author information is \\ available at the end of the article
}

\begin{abstract}
Understanding the generation mechanism of the heat flux is essential for the design of hypersonic vehicles. We proposed a novel formula to decompose the heat flux coefficient into the contributions of different terms by integrating the conservative equation of the total energy. The reliability of the formula is well demonstrated by the direct numerical simulation results of a hypersonic transitional boundary layer. Through this formula, the exact process of the energy transport in the boundary layer can be explained and the dominant contributors to the heat flux can be explored, which are beneficial for the prediction of the heat and design of the thermal protection devices.
\end{abstract}

Keywords: Heat flux, Hypersonic boundary layer, Direct numerical simulation

\section{Introduction}

The heat transfer prediction is of great importance for hypersonic vehicles. When the transition or turbulence happens, the accurate prediction becomes even more challenging. Therefore, exploring the generation mechanism of the heat flux has drawn wide attention. Many efforts have been put into understanding the generation mechanism of the heat flux, which provides important guidance for the design of the thermal protection system and the thermal management [1].

Some efforts took advantage of the similarities between the generation of friction and heat flux, and constructed the Reynolds analogy [2] $R a=2 S t / C_{f}$ to connect friction with heat flux, where $S t$ is the Stanton number, $C_{f}$ the skin friction coefficient. Hopkins and Inouye [3] predicted the surface heat flux of the hypersonic boundary layer with $R a=1$. However, the accuracy of this correlation decreases significantly on a cold wall at high Mach numbers. More intrinsic mechanisms should be considered. Huang et al. [4] derived the formula $q_{w}=-u_{b} \tau_{w}$ by the assumption that the heat transfer into the walls equals the total pressure work done across the channel. Chen et al. [5] established the exact relations for the skin friction with other dynamic and kinetic quantities, and they found these relations revealed that the skin friction is intrinsically coupled with the surface temperature through the heat flux. Abe and Antonia [6] proposed a simple relation between the scalar dissipation rate and the wall heat transfer coefficient for the channel flow. Kim et al. [7] proposed a direct approach for the time-

(c) The Author(s). 2021 Open Access This article is licensed under a Creative Commons Attribution 4.0 International License, which permits use, sharing, adaptation, distribution and reproduction in any medium or format, as long as you give appropriate credit to the original author(s) and the source, provide a link to the Creative Commons licence, and indicate if changes were made. The images or other third party material in this article are included in the article's Creative Commons licence, unless indicated otherwise in a credit line to the material. If material is not included in the article's Creative Commons licence and your intended use is not permitted by statutory regulation or exceeds the permitted use, you will need to obtain permission directly from the copyright holder. To view a copy of this licence, visit http://creativecommons.org/licenses/by/4.0/. 
dependent heat flux by assuming the temperature approximated as a third-order polynomial of position. Ebadi et al. [8] obtained the wall heat flux by a triple integration of the Reynolds averaged energy equation for the two-dimensional incompressible turbulent boundary layer. Fukagata et al. [9] studied the relationship of the wall heat flux and the Nusselt number in the incompressible channel. And the similar relation was obtained by Liu [10] when studying the compressible pipe flow. Ghosh [11] proposed that the wall heat flux can be expressed by the sum of the integrations of different viscous terms in the incompressible turbulent channel and pipe flow. Zhang and Xia [12] further proposed a formula to assess the contributions of the viscous stresses to the heat transfer in a turbulent channel flow by the method of Fukagata et al. [13], but the turbulent stresses were missing in their expression due to the simplification assumptions used in the channel flow.

Previous studies have not built the relation of the generation mechanism of the heat flux to the dynamic energy transport of the compressible boundary layer. Therefore, a more reliable and accurate method to gain insights into the generation mechanism should be derived. In the paper, we propose a new decomposition formula for the wall heat flux by integrating the conservation equation of total energy, which can explain the energy transport process in the boundary layer and reveal the main factors affecting the wall heat flux. As far as the authors' knowledge, this is the first decomposition formula proposed for the wall heat flux of the compressible boundary layer. The new formula is derived by the method of Renard et al. [14], which is more physical and feasible than those [11, 12] based on the method of Fukagata et al. [13] It serves an insight into the complex transport processes of the wall heat flux, and helps us find the key factors.

The work of this paper is organized as follows. In section 2, we describe numerical methods and case setup in brief. In section 3 , the detailed derivation of the decomposition formula is presented. In section 4, the validation of the DNS of a hypersonic transitional boundary layer is performed, and the proposed decomposition formula is applied to analyze the heat flux. Finally, some conclusions are drawn in section 5.

\section{Numerical methods and case setup}

To analyze the heat flux decomposition of the hypersonic transitional boundary layer, a direct numerical simulation is performed. The compressible Navier-Stokes equations in the curvilinear coordinate are adopted as governing equations.

$$
\begin{aligned}
& \frac{\partial \rho}{\partial t}+\frac{\partial \rho u_{j}}{\partial x_{j}}=0 \\
& \frac{\partial \rho u_{i}}{\partial t}+\frac{\partial \rho u_{i} u_{j}}{\partial x_{j}}+\frac{\partial p \delta_{i j}}{\partial x_{j}}=\frac{\partial \sigma_{i j}}{\partial x_{j}} \\
& \frac{\partial \rho e}{\partial t}+\frac{\partial u_{j}(\rho e+p)}{\partial x_{j}}=\frac{\partial u_{j} \sigma_{i j}}{\partial x_{j}}+\frac{\partial q_{j}}{\partial x_{j}}
\end{aligned}
$$

Where $u_{1}, u_{2}$ and $u_{3}$ are streamwise, normal and spanwise velocities, respectively. And $p$ and $\rho$ are pressure and density. The expressions of $\rho e, \sigma_{i j}$, and $q_{j}$ are defined as

$$
\rho e=\frac{p}{\gamma-1}+\frac{1}{2} \rho\left(u^{2}+v^{2}+w^{2}\right)
$$




$$
\begin{aligned}
\sigma_{i j} & =\frac{\mu}{R e_{\infty}}\left[\left(\frac{\partial u_{i}}{\partial x_{j}}+\frac{\partial u_{j}}{\partial x_{i}}\right)-\frac{2}{3} \delta_{i j} \frac{\partial u_{k}}{\partial x_{k}}\right] \\
q_{j} & =\frac{\mu}{P r M a_{\infty}^{2} R e_{\infty}(\gamma-1)} \frac{\partial T}{\partial x_{j}} .
\end{aligned}
$$

The usual indicial notation is used. Prandtl number $\operatorname{Pr}$ is set as 0.72 and the specific heat ratio $\gamma$ is 1.4. The dynamic viscosity $\mu$ is obtained by using Sutherland's law. $M a_{\infty}$ is the freestream Mach number and $R e_{\infty}$ is the Reynolds number. The working fluid is air, and the gas model is assumed to be the perfect gas model.

An in-house code with high-order schemes is employed to perform the DNS. This code has been applied in many DNS simulations of compressible turbulent cases, including compressible homogeneous turbulence [15], turbulent boundary layer [15] and shock wave/boundary layer interaction [16-18]. The accuracy and robustness have been well validated. The hybrid optimized WENO scheme is adopted to discretize the inviscid fluxes with a novel discontinuity detector [19]. In the regions with discontinuities, a seventh-order WENO scheme [20] is activated and in the smooth region, a fourth-order bandwidth-optimized upwind-biased scheme [19] is used to resolve the small structures in the turbulent boundary layer. The viscous terms are discretized by the fourth-order central scheme. The thirdorder TVD Runge-Kutta method is adopted as the temporal algorithm. $M a_{\infty}$ is set as 6.0, and $R e_{\infty}$ is 12,000 which is based on the millimetre and freestream parameters. The freestream temperature is $65 \mathrm{~K}$ and the wall temperature is $305 \mathrm{~K}$. The millimetre is adopted as the reference length.

The sketch of the computational domain is presented in Fig. 1. The lengths in the streamwise, normal and spanwise direction are $L_{x}=416, L_{y}=35$ and $L_{z}=14$, respectively. The computational domain is discretized with $N_{x} \times N_{y} \times N_{z}=1151 \times$ $320 \times 149$ points. The grid points are equally spaced in the spanwise direction, and clustered near the wall in the normal direction. The grid spacing normalized by the wall unit in the three directions is $d x^{+}=7.9, d y_{w}^{+}=0.36$ and $d z^{+}=3.4$, respectively. A hypersonic laminar profile is fixed at the inlet. The outlet boundary condition is a supersonic outflow with a sponge layer to further suppress the disturbances originated from the outlet. The upper boundary is a non-reflective condition. And an isothermal nonslip condition is applied at the wall. The periodical conditions are set on both sides in the spanwise direction. To trigger a bypass transition, a blow and suction forcing method is employed, which sets a normal

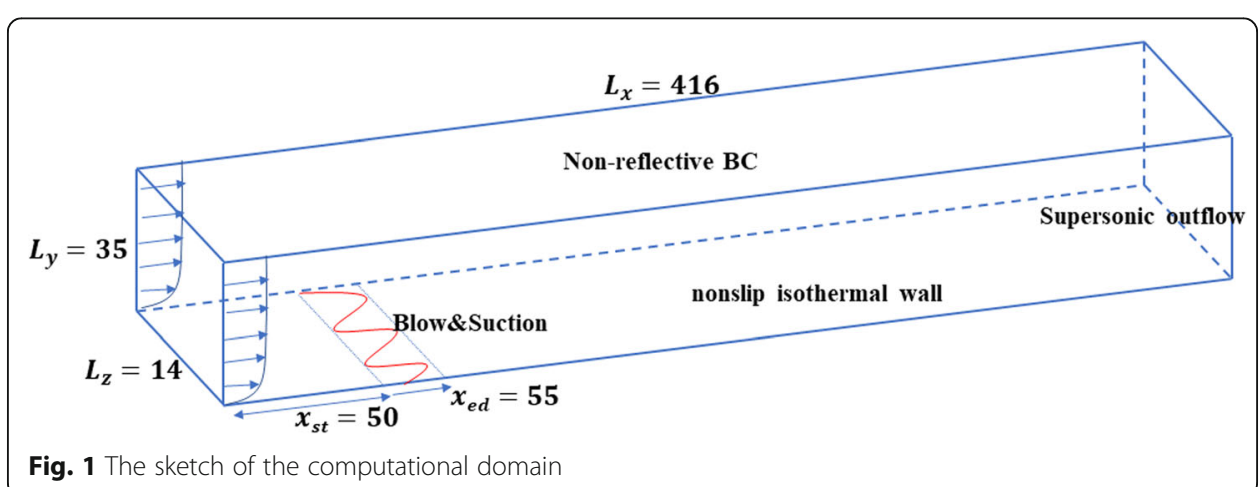


velocity disturbance $v_{n}=A f(x) \cos \left(2 \pi \lambda_{z} / L_{z}\right)$ at the wall, where $f(x)$ is used to control the streamwise extent of force [21], $\lambda_{z}$ is the spanwise wavelength and is set as 4.0 in the present simulation. The forcing region ranges from $x=50$ to $x=55$.

\section{Derivation of the decomposition formula}

Under the assumption of homogeneity in the spanwise direction and nonslip condition at the wall, the time-averaged conservative equations for the specific total energy can be expressed as,

$$
\bar{\rho} \frac{D \tilde{E}}{D t}+\frac{\partial \bar{p} \tilde{u}_{j}}{\partial x_{j}}=\frac{\partial}{\partial x_{j}}\left(q_{L, j}+q_{T, j}+D_{j}+T_{j}+M S_{j}+R S_{j}\right)
$$

when $\phi$ is an arbitrary variable, $\bar{\phi}$ denotes Reynolds average, $\tilde{\phi}$ Favre average and $\phi "$ is the fluctuations concerning the Favre average. $\tilde{E}$ denotes the specific total energy, $q_{L, j}$ the heat conduction, $q_{T, j}$ the turbulent transport of heat, $D_{j}$ the molecular diffusion, $T_{j}$ the turbulent transport of turbulent kinetic energy (TKE), $M S_{j}$ work by the molecular stresses and $R S_{j}$ work by Reynold stresses. In Table 1, the specific expressions of the terms at the right hand of Eq. (5) are presented. More details about Eq. (5) can be found in the book of Wilcox [22].

The work of Renard [14] and Li [23] about skin friction decomposition provides good hints for building the integration relation of heat flux. In order to isolate the effects of the energy transport terms, the absolute reference frame is employed. The reference absolute frame is attained by assuming that the wall moves at the speed $U_{\infty}$. The expressions of the time $t_{a}$, coordinates $x_{a}, y_{a}$, velocities $u_{a}, v_{a}$, pressure $p_{a}$ and the density $\rho_{a}$ in the reference absolute frame satisfy

$$
\begin{gathered}
t_{a}=t, \quad x_{a}=x-U_{\infty} t, \quad y_{a}=y, \\
u_{a}=u-U_{\infty}, \quad v_{a}=v, \\
p_{a}=p, \quad \rho_{a}=\rho
\end{gathered}
$$

where the subscript ' $a$ ' represents 'absolute' variables under the reference frame. By substituting Eq. (6) into Eq. (5), Eq. (5) takes the form

Table 1 Expressions for the terms at the right hand of Eq. (5)

\begin{tabular}{ll}
\hline Heat conduction & $q_{L, j}=\overline{k \partial T / \partial x_{j}}$ \\
Turbulent transport of heat & $q_{T, j}=-\overline{\rho u_{j}^{\prime \prime} h^{\prime \prime}}$ \\
Molecular diffusion & $D_{j}=\overline{u_{k}^{\prime \prime} \sigma_{k j}}$ \\
Turbulent transport of TKE & $T_{j}=\overline{\rho u_{j}^{\prime \prime} u_{k}^{\prime \prime} u_{k}^{\prime \prime} / 2}$ \\
Work by molecular stresses & $M S_{j}=\tilde{u}_{k} \bar{\sigma}_{k, j}$ \\
Work by Reynolds stresses & $R S_{j}=-\tilde{u}_{k} \overline{\rho u_{k}^{\prime \prime} u_{j}^{\prime \prime}}$
\end{tabular}




$$
\begin{aligned}
& \bar{\rho}_{a} \frac{D \tilde{E}_{a}}{D t_{a}}+\frac{\partial\left(\bar{p}_{a} \tilde{u}_{a}\right)}{\partial\left(x_{a}+U_{\infty} t_{a}\right)}+\frac{\partial\left(\bar{p}_{a} \tilde{v}_{a}\right)}{\partial y_{a}} \\
& =\frac{\partial}{\partial y_{a}}\left(q_{L, y, a}+q_{T, y, a}+D_{y, a}+T_{y, a}+M S_{y, a}+R S_{y, a}\right) \\
& +\frac{\partial}{\partial\left(x_{a}+U_{\infty} t_{a}\right)}\left(q_{L, x, a}+q_{T, x, a}+D_{x, a}+T_{x, a}+M S_{x, a}+R S_{x, a}\right)
\end{aligned}
$$

By multiplying $\tilde{u}_{a}$ at both sides of Eq. (7), combining the continuity equation and moving the heat fluxes in the normal direction to the left side and all the other terms to the right, the following equation could be obtained,

$$
\begin{aligned}
& \tilde{u}_{a} \frac{\partial}{\partial y_{a}}\left(q_{L, y, a}+q_{T, y, a}\right) \\
& =\tilde{u}_{a} \bar{\rho}_{a} \frac{D \tilde{E}_{a}}{D t_{a}}+\tilde{u}_{a} \frac{\partial\left(\bar{p}_{a} \tilde{u}_{a}\right)}{\partial\left(x_{a}+U_{\infty} t_{a}\right)}+\tilde{u}_{a} \frac{\partial\left(\bar{p}_{a} \tilde{v}_{a}\right)}{\partial y_{a}} \\
& -\tilde{u}_{a} \frac{\partial}{\partial y_{a}}\left(D_{y, a}+T_{y, a}+M S_{y, a}+R S_{y, a}\right) \\
& -\tilde{u}_{a} \frac{\partial}{\partial\left(x_{a}+U_{\infty} t_{a}\right)}\left(q_{L, x, a}+q_{T, x, a}+D_{x, a}+T_{x, a}+M S_{x, a}+R S_{x, a}\right)
\end{aligned}
$$

Integrating Eq. (8) from the wall to the farfield, the heat flux on the wall $(y=0)$ can be expressed as the following form.

$$
\begin{aligned}
& \left.q_{L, y, a}\right|_{y=0}= \\
& \frac{1}{U_{\infty}} \int_{0}^{\infty}\left[\left(q_{L, y, a}+q_{T, y, a}\right) \frac{\partial \tilde{u}_{a}}{\partial y_{a}}\right] d y \\
& -\frac{1}{U_{\infty}} \int_{0}^{\infty}\left[\tilde{u}_{a} \frac{\partial}{\partial y_{a}}\left(D_{y, a}+T_{y, a}+M S_{y, a}+R S_{y, a}\right)\right] d y \\
& +\frac{1}{U_{\infty}} \int_{0}^{\infty}\left[\tilde{u}_{a} \bar{\rho}_{a} \frac{D \tilde{E}_{a}}{D t_{a}}+\tilde{u}_{a} \frac{\partial\left(\bar{p}_{a} \tilde{u}_{a}\right)}{\partial\left(x_{a}+U_{\infty} t_{a}\right)}+\tilde{u}_{a} \frac{\partial\left(\bar{p}_{a} \tilde{v}_{a}\right)}{\partial y_{a}}\right] d y \\
& -\frac{1}{U_{\infty}} \int_{0}^{\infty}\left[\tilde{u}_{a} \frac{\partial}{\partial\left(x_{a}+U_{\infty} t_{a}\right)}\left(q_{L, x, a}+q_{T, x, a}+D_{x, a}+T_{x, a}+M S_{x, a}+R S_{x, a}\right)\right] d y
\end{aligned}
$$

Finally, Eq. (9) can be transformed into the initial frame and the heat flux coefficient is obtained by introducing the definition of the coefficient $C_{h}=\left.\frac{1}{\rho_{\infty} U_{\infty}^{3}} \kappa \frac{\partial T}{\partial y}\right|_{y=0}$,

$$
\begin{aligned}
& C_{h, \text { decomp }}=\underbrace{\frac{1}{\rho_{\infty} U_{\infty}^{4}} \int_{0}^{\infty} q_{L, y} \frac{\partial \tilde{u}}{\partial y} d y}_{C_{h, 1}}+\underbrace{\frac{1}{\rho_{\infty} U_{\infty}^{4}} \int_{0}^{\infty} q_{T, y} \frac{\partial \tilde{u}}{\partial y} d y}_{C_{h, 2}}-\underbrace{\frac{1}{\rho_{\infty} U_{\infty}^{4}} \int_{0}^{\infty}\left(\tilde{u}-U_{\infty}\right) \frac{\partial D_{y}}{\partial y} d y}_{C_{h, 3}} \\
& -\underbrace{\frac{1}{\rho_{\infty} U_{\infty}^{4}} \int_{0}^{\infty}\left(\tilde{u}-U_{\infty}\right) \frac{\partial T_{y}}{\partial y} d y}_{C_{h, 4}}-\underbrace{\frac{1}{\rho_{\infty} U_{\infty}^{4}} \int_{0}^{\infty}\left(\tilde{u}-U_{\infty}\right) \frac{\partial M S_{y}}{\partial y} d y}_{C_{h, 5}}-\underbrace{\frac{1}{\rho_{\infty} U_{\infty}^{4}} \int_{0}^{\infty}\left(\tilde{u}-U_{\infty}\right) \frac{\partial R S_{y}}{\partial y} d y}_{C_{h, 6}} \\
& +\underbrace{\frac{1}{\rho_{\infty} U_{\infty}^{4}} \int_{0}^{\infty}\left(\tilde{u}-U_{\infty}\right)\left[\bar{\rho} \frac{D \tilde{E}}{D t}+\frac{\partial \bar{p} \tilde{u}}{\partial x}+\frac{\partial \bar{p} \tilde{v}}{\partial y}-\frac{\partial}{\partial x}\left(q_{L, x}+q_{T, x}+D_{x}+T_{x}+M S_{x}+R S_{x}\right)\right] d y}_{C_{h, 7}}
\end{aligned}
$$

In Eq. (10), the heat flux coefficient $C_{h, \text { decomp }}$ has been decomposed into seven parts, i.e., $C_{h, 1}$ to $C_{h, 7}$. The first part $C_{h, 1}$ represents the contribution of heat conduction, the 
second part $C_{h, 2}$ the turbulent transport of heat, the third part $C_{h, 3}$ the normal component of the molecular diffusion, the fourth part $C_{h, 4}$ the turbulent transport of turbulent kinetic energy (TKE), the fifth part $C_{h, 5}$ the work by the molecular stresses and the sixth part $C_{h, 6}$ the work by Reynolds stresses. And the last part $C_{h, 7}$ contains the streamwise heterogeneity, pressure work and the variation of the specific total energy with time.

\section{Decomposition results of the wall heat flux}

In this section, the reliability and accuracy of the new decomposition formula will be demonstrated by the DNS results of a hypersonic transitional boundary layer. The contributions of different energy transport processes such as the heat conduction, the turbulent transport of heat, the work done by molecular stresses will be calculated. Moreover, the key normal locations where the structures impact the wall heat will be assessed by the integrand functions of these contributions.

\subsection{DNS results of the hypersonic transitional boundary layer}

The instantaneous vortical structures displayed by $Q$ criterion [24] are presented in Fig. 2. The transition process can be observed. Three streamwise locations (P1, P2 and P3) are chosen to validate the decomposition results, which are marked by shade planes. In Fig. 3, the mean streamwise velocity after van Driest transformation at P3 is presented and compared with DNS results of hypersonic results by Priebe [25]. Good agreement is obtained. The discrepancy in the outer part of the boundary layer is caused by the different friction velocity at the wall. In Fig. 4, the Reynolds stresses normalized by the friction velocity are presented. Several supersonic and hypersonic results [26-28] are also displayed. The information of the setup of reference simulations is presented in Table 2 , where $\delta_{\text {ref }}$ is the nominal boundary layer thickness in the fully developed region. The present data generally agree with those in the reference results. These comparisons demonstrate that the performance of our present direct simulation is acceptable, and the obtained data can be used for decomposition analysis.

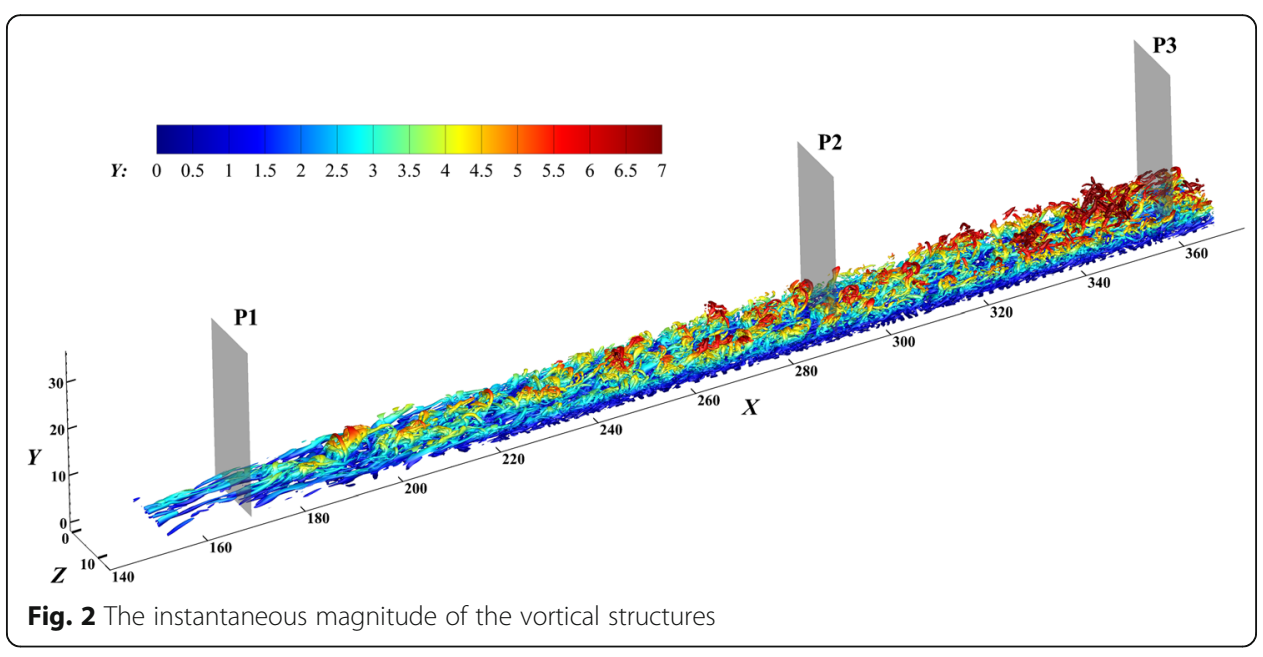




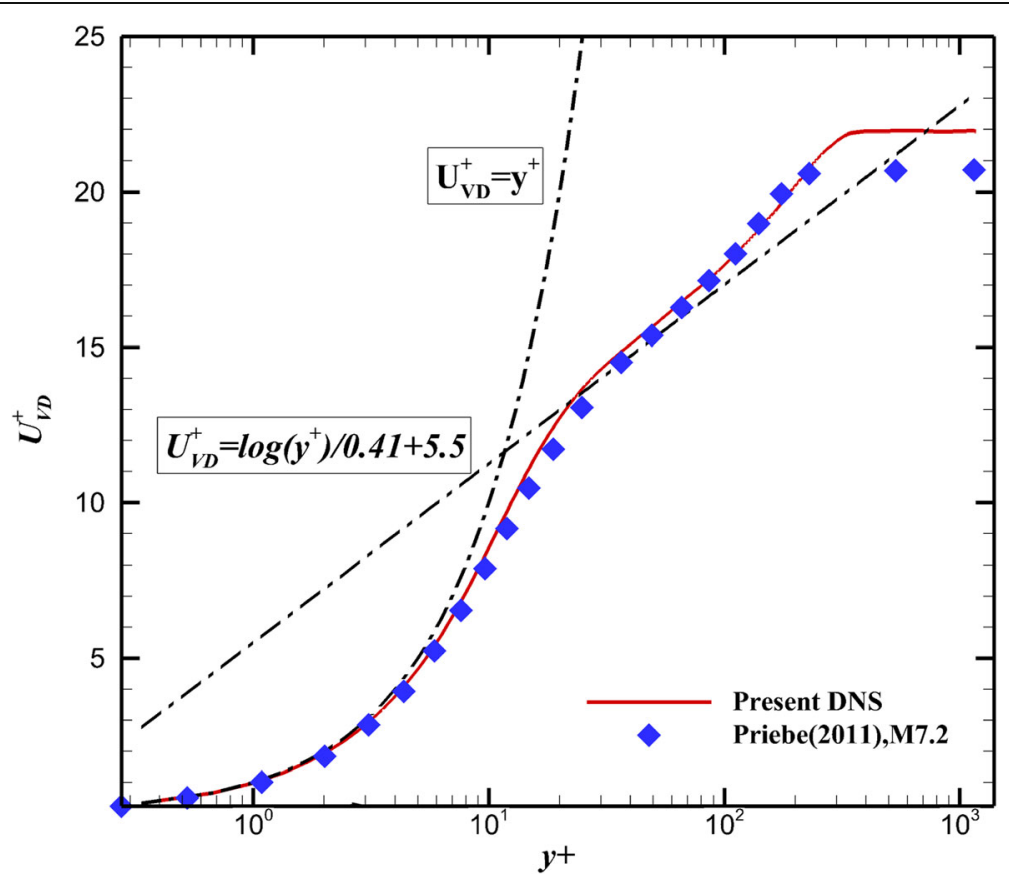

Fig. 3 van Driest transformed mean streamwise velocity

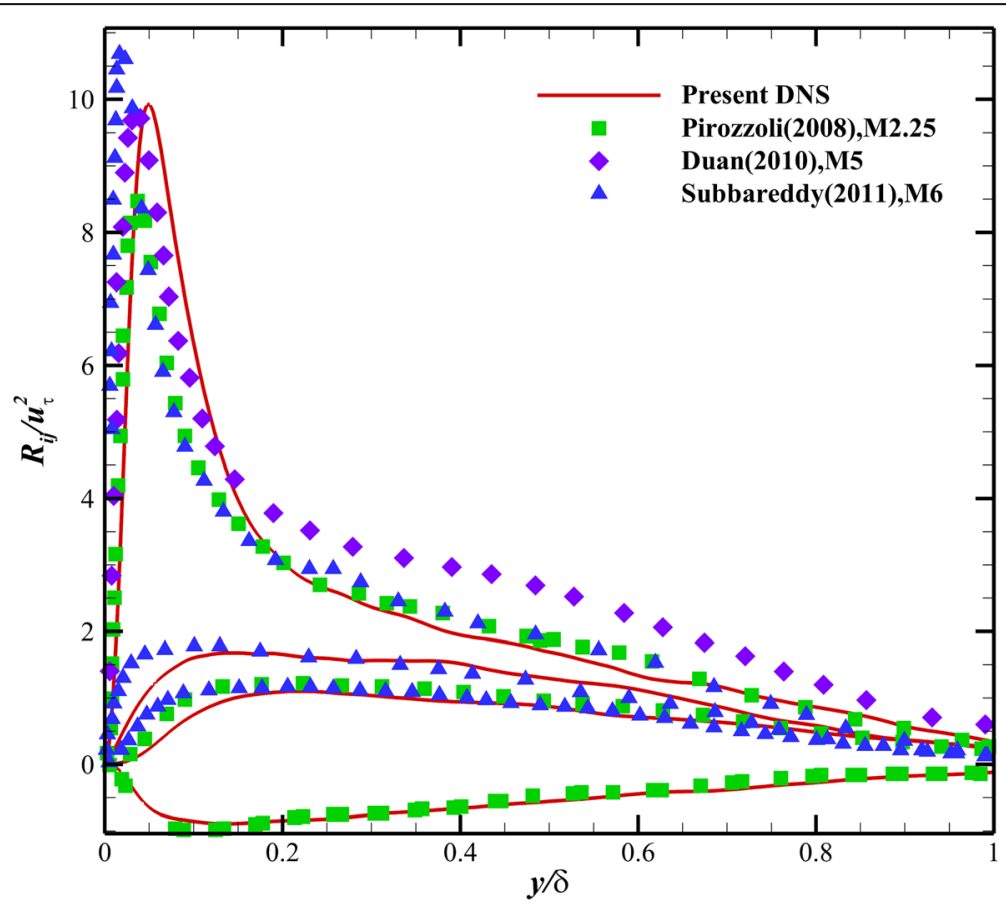

Fig. 4 Streamwise turbulent intensity normalized by Morkovin transformed velocity 
Table 2 Setup of the reference simulations

\begin{tabular}{|c|c|c|c|c|}
\hline & $M a$ & $\left(L_{x}, L_{y}, L_{z}\right)$ & $\left(N_{x}, N_{y}, N_{z}\right)$ & $\left(\Delta x^{+}, \Delta y^{+}, \Delta z^{+}\right)$ \\
\hline Priebe (2011) & 7.2 & $\left(27.0 \delta_{\text {ref }}, 14.2 \delta_{\text {ref }} 10.0 \delta_{\text {ref }}\right)$ & $(840,150,768)$ & $(7.5,0.3,3.0)$ \\
\hline Pirozzoli (2008) & 2.0 & $\left(25.0 \delta_{\text {ref }}, 3 \delta_{\text {ref }}, 3.7 \delta_{\text {ref }}\right)$ & $(1809,180,225)$ & $(4.5,0.95,4.5)$ \\
\hline Duan (2010) & 5.0 & $\left(7.5 \delta_{\text {ref, }} 16.7 \delta_{\text {ref, }} 1.9 \delta_{\text {ref }}\right)$ & $(384,110,256)$ & $(7.7,0.33,2.9)$ \\
\hline Subbareddy (2011) & 6.0 & $N A$ & $(5400,175,175)$ & $(30.0,0.5,25.0)$ \\
\hline Present DNS & 6.0 & $\left(64.0 \delta_{\text {ref }}, 5.4 \delta_{\text {ref }}, 2.2 \delta_{\text {ref }}\right)$ & $(1151,320,149)$ & $(7.9,0.36,3.4)$ \\
\hline
\end{tabular}

\subsection{Contributions of different energy transport processes}

The contributions of different energy transport processes to the wall heat flux at different streamwise locations, e.g. P1-P3 are calculated according to Eq. (10). The ratios of the contributions of the terms in Eq. (10) to the reconstructed heat flux coefficient $C_{h \text {,decom }}$ and the relative errors are presented in Table 3 . The relative error is defined as Error $=\left(C_{h \text {,decom }}-C_{h, 0}\right) / C_{h, 0} \times 100 \%$, where $C_{h, 0}$ is the time-averaged heat flux coefficient. The relative errors are very small in both transitional and turbulent regions. In Table 3 , it is found that the work done by the molecular stresses $\left(C_{h, 5}\right)$ and Reynolds stresses $\left(C_{h, 6}\right)$ plays dominant roles in heat production. The sum of the contributions of these two terms takes over 2.7 times of $C_{h, 0}$ in the transition region and 1.9 times in the turbulent region. The contributions of the heat conduction $\left(C_{h, 1}\right)$ and the turbulent transport of heat $\left(C_{h, 2}\right)$ are negative, which indicates both processes act as the transporters of the heat and carry the extra heat to the outer regions of the boundary layer. The magnitude of $C_{h, 1}$ and $C_{h, 5}$ gets greater in the turbulent region due to the large gradient of the profiles. On the other hand, $C_{h, 2}$ and $C_{h, 6}$ in the transitional region are much greater than those in the turbulent region, because of the quick nonlinear development of the structures.

The trends of the contributions can be further assessed when more sample locations are considered. The trends of the contributions in the transition process are presented in Fig. 5. In addition, the instantaneous density gradient and the time-averaged heat flux coefficient are also presented to show the overall evolution procedure. The symbols in the line of $C_{h, i}$ indicate different sample locations (from left to right labelled as $\mathrm{X} 0$ to X10). The first sample point (X0) is located in the laminar region; therefore, the work of the molecular stress and the heat conduction is the main factor affecting the wall heat. After entering the transition region, the effects of the Reynold stresses become dominant. Moreover, a rapid variation of $C_{h, 6}$ is observed in the transition region due to the nonlinear development of the coherent structures. While in the turbulent region, an equilibrium state is reached and a smooth variation is obtained. The trends of contributions of the heat conduction $C_{h, 1}$ and the molecular stresses $C_{h, 5}$ are very smooth in both transition and turbulent regions, which indicate they are not sensitive to the change of the turbulent fluctuations. In addition, the effects of the molecular

Table 3 Contributions of the terms in Eq. (10)

\begin{tabular}{lllllllllll}
\hline & $C_{h, 1} / C_{h, \text { decom }}$ & $C_{h, 2} / C_{h, \text { decom }}$ & $C_{h, 3} / C_{h, \text { decom }}$ & $C_{h, 4} / C_{h, \text { decom }}$ & $C_{h, 5} / C_{h, \text { decom }}$ & $C_{h, 6} / C_{h, \text { decom }}$ & $C_{h, 7} / C_{h, \text { decom }}$ & $C_{h, 0}$ & $C_{h, \text { decom }}$ & Err \\
\hline P1 & -0.111 & -1.379 & 0.018 & -0.084 & 0.478 & 2.437 & -0.359 & $1.195 \mathrm{E}-4$ & $1.183 \mathrm{E}-4$ & $1.00 \%$ \\
P2 & -0.213 & -0.785 & 0.012 & -0.072 & 0.673 & 1.299 & 0.086 & $1.825 \mathrm{E}-4$ & $1.819 \mathrm{E}-4$ & $0.33 \%$ \\
P3 & -0.208 & -0.713 & 0.015 & -0.054 & 0.662 & 1.200 & 0.098 & $1.616 \mathrm{E}-4$ & $1.620 \mathrm{E}-4$ & $0.25 \%$ \\
\hline
\end{tabular}




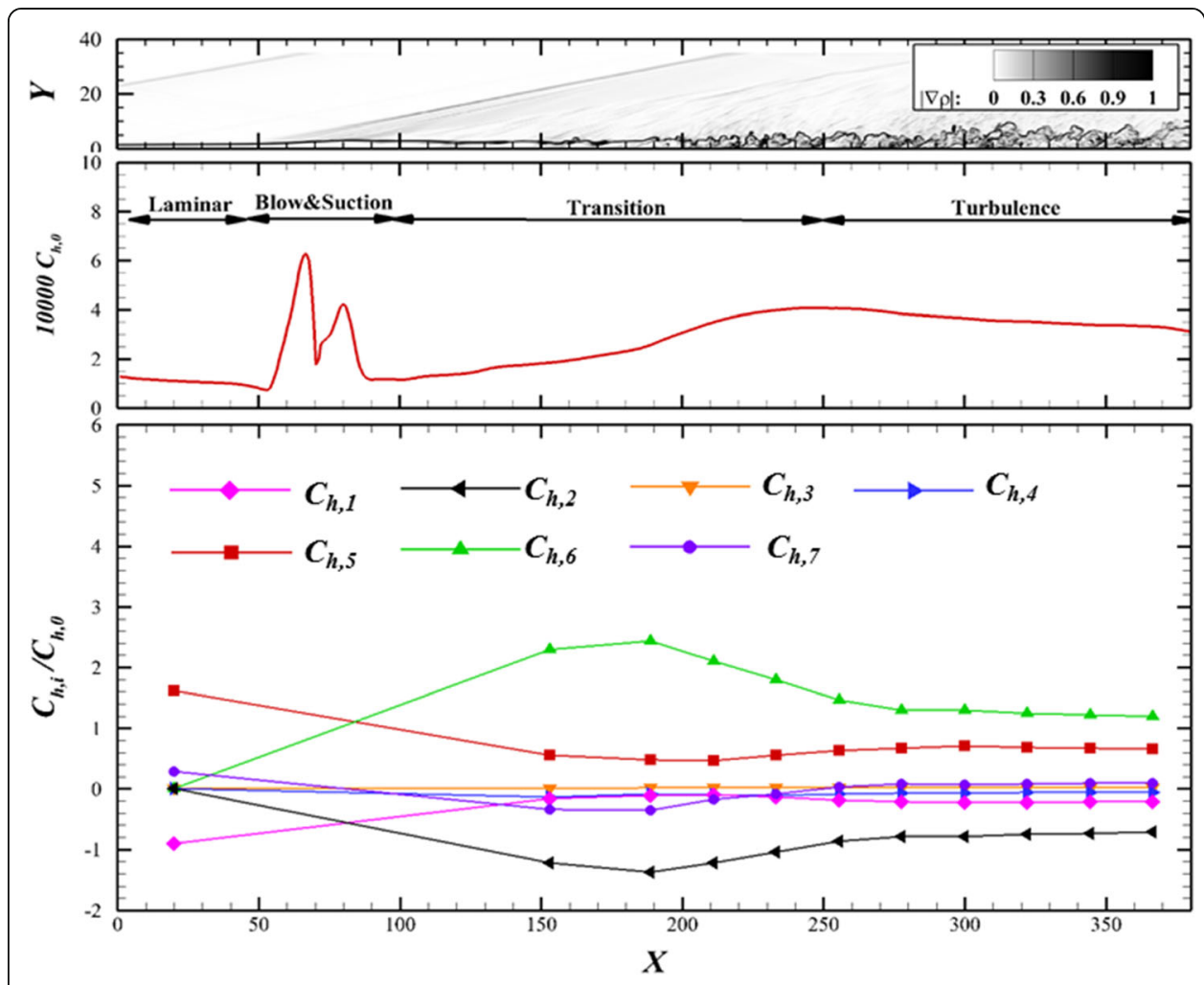

Fig. 5 The streamwise trends of the contributions of the terms of heat flux decomposition

diffusion $\left(C_{h, 3}\right)$, the turbulent transport of TKE $\left(C_{h, 4}\right)$ and the streamwise heterogeneity $\left(C_{h, 7}\right)$ are very small during the whole transition.

\subsection{Key normal locations affecting the wall heat}

The key normal locations where the structures affect the wall heat can be attained by analyzing the integrand functions of the terms in Eq. (10). Two main transporters, i.e., the heat conduction $\left(C_{h, 2}\right)$ and the turbulent transport $\left(C_{h, 3}\right)$ and two main contributors, i.e., the work by molecular stresses $\left(C_{h, 5}\right)$ and Reynolds stresses $\left(C_{h, 6}\right)$ are analyzed in this section.

The integrand functions of the heat conduction $\left(C_{h, 2}\right)$ and the turbulent transport $\left(C_{h, 3}\right)$ are presented in Fig. 6 . The lines in the figure denote the locations in the transitional region and the symbols are the locations in the turbulent region. The contribution of $C_{h, 2}$ is positive when the normal locations are near the wall due to the positive temperature gradient. When $y^{+}$reaches 10 , a valley is observed, which is formed by the decrease of the temperature after the peak of the mean temperature. Compared with the integrand function of $C_{h, 2}$, two valleys are observed in the integrand functions of $C_{h, 3}$. The first valley is located at $y^{+} \approx 65$, the magnitude of the valley reduces and finally disappears as the locations move downstream. The second valley is located at around $y^{+}=10$, which corresponds to the buffer layer of the turbulent boundary layer. The valley will get larger as the locations move downstream. 


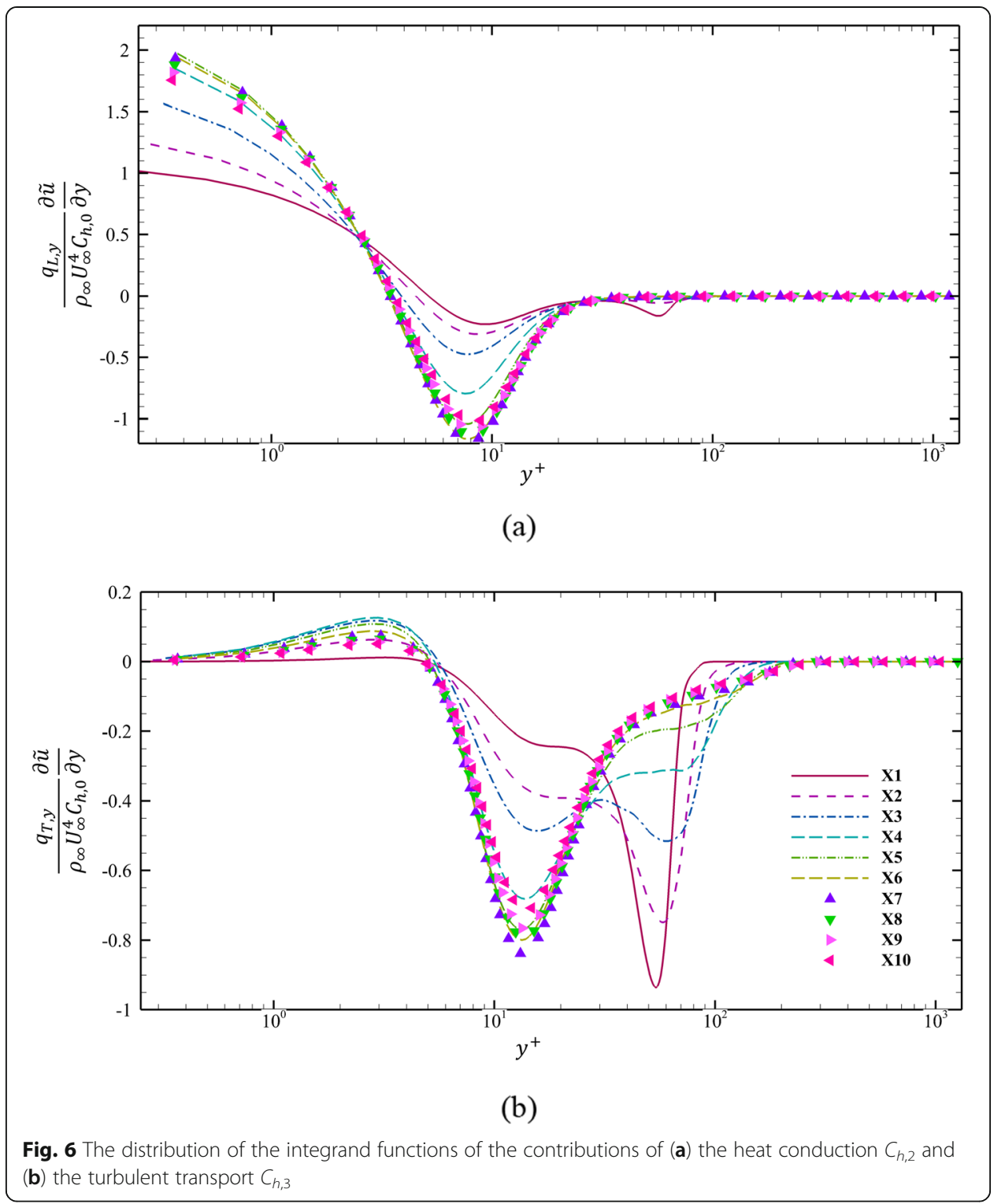

The key normal locations of the work by molecular stresses $\left(C_{h, 5}\right)$ and Reynolds stresses $\left(C_{h, 6}\right)$ are analyzed in Fig. 7 . The positive contribution of $C_{h, 5}$ is mainly located in the regions near the wall. As the normal location increases, the integrand function will decrease and a valley is formed around $y^{+}=10$. Meanwhile, a peak is observed around $y^{+}=10$ in the integrand function of contribution of $C_{h, 6}$. The peak is located in the buffer layer of the boundary layer. In addition, in the transition process, the magnitude of the peak will get larger as the streamwise distance increases.

\section{Conclusions}

In this paper, we proposed a new decomposition formula for the wall heat flux. And the performance of the formula has been well demonstrated by DNS results of a hypersonic transitional boundary layer. 


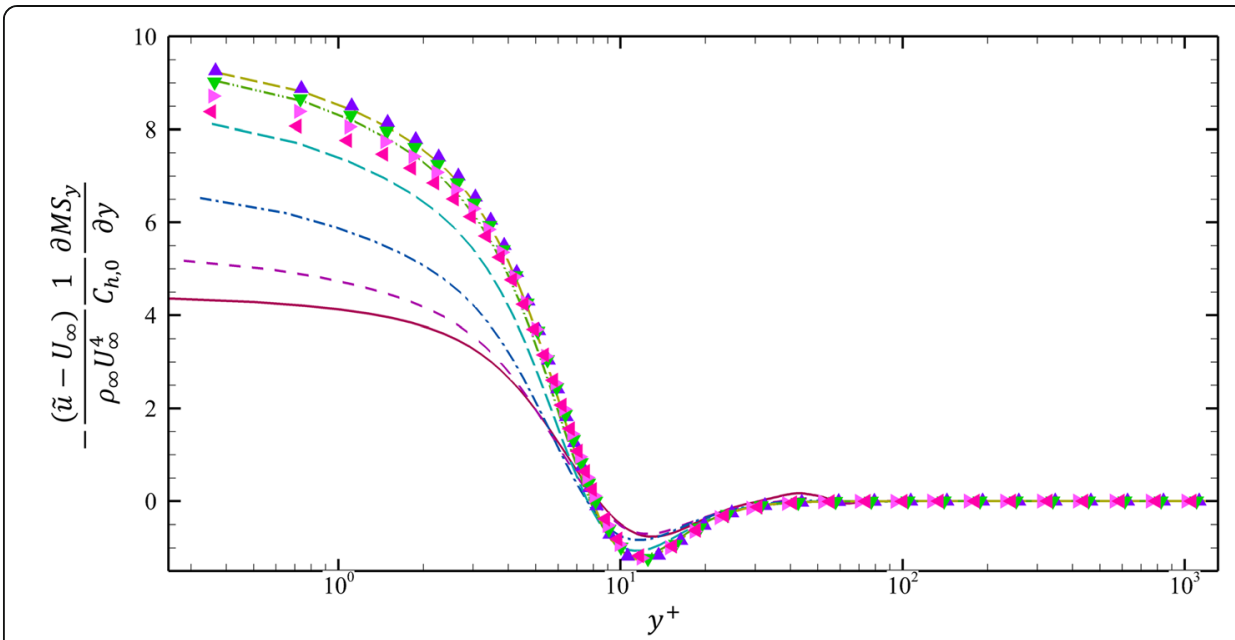

(a)

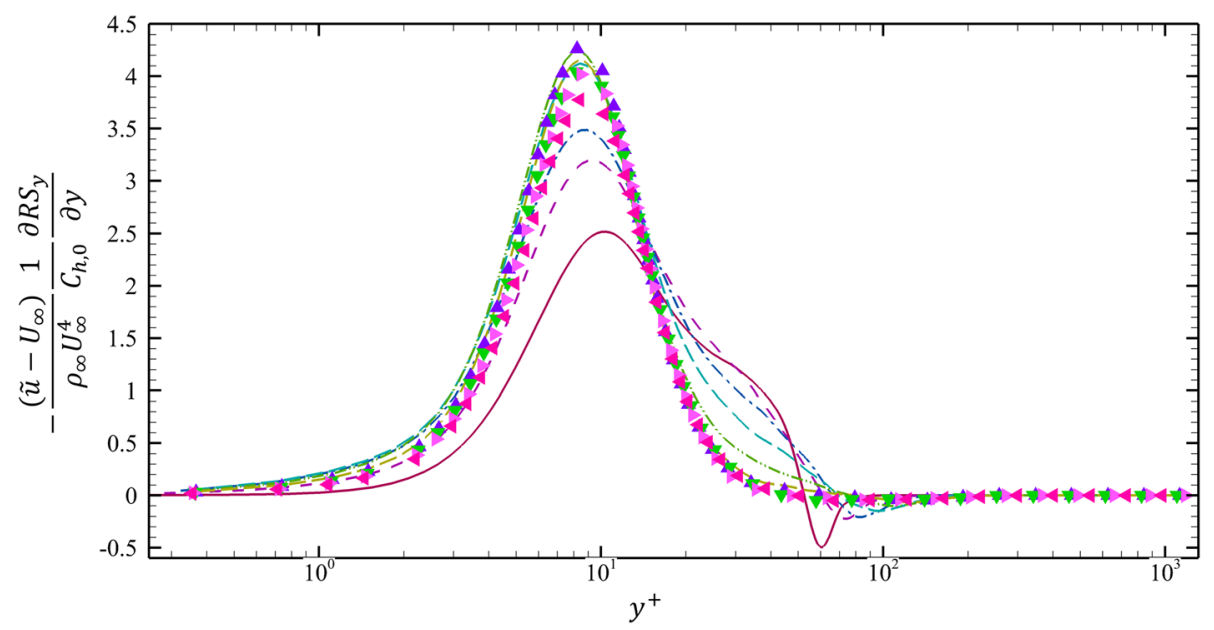

(b)

Fig. 7 The distribution of the integrand of the contribution of work done by (a) molecular stresses $C_{h, 5}$ and (b) Reynolds stresses $C_{h, 6}$

(1) Through this formula, the wall heat flux can be decomposed into contributions of seven terms, i.e., the heat conduction, turbulent transport of heat, molecular diffusion, turbulent transport of TKE, molecular stresses, the Reynolds stresses and the streamwise heterogeneity.

(2) The contributions of each term can be calculated quantitatively. For the present case, it is found that the heat flux produced by the work done by Reynolds stresses and molecular stresses is much higher than the time-averaged heat flux on the wall. The heat conduction and turbulent convection will carry the extra heat into the outer part of the boundary layer.

(3) The structures in the buffer layer $\left(y^{+}=10\right)$ play a dominant role in the production and transfer of the heat flux for the present simulation. Moreover, the contributions of the heat conduction and the molecular stresses are mainly affected by the gradient of the temperature. 
(4) As the assumption used in the derivation is only the spanwise homogeneity and nonslip wall, this formula can be applied in the analysis of the heat transfer of a hypersonic transitional/turbulent boundary layer at high Mach numbers, which can be employed to identify the main factors affecting the wall heating and provide good guidance for the design of the thermal protection system.

\section{Acknowledgements}

Not applicable.

\section{Authors' contributions}

The major contribution of this manuscript is due to the first author, while the second author and the third author give important suggestions on the derivation of the formulas. The author(s) read and approved the final manuscript.

\section{Funding}

This work was supported by the National Key Research and Development Program of China (Grant No. 2019YFA0405201, No. 2019YFA0405300), the National Natural Science Foundation of China (Grant No.11802324), and the National Numerical Windtunnel Project.

\section{Availability of data and materials}

The data that support the findings of this study are available from the corresponding author upon reasonable request.

\section{Declarations}

\section{Competing interests}

The authors declare that they have no competing interests.

\section{Author details}

${ }^{1}$ State Key Laboratory of Aerodynamics, Mianyang 621000, Sichuan, China. ${ }^{2}$ Computational Aerodynamics Institute, China Aerodynamics Research and Development Center, Mianyang 621000, Sichuan, China.

Received: 28 June 2021 Accepted: 9 August 2021

Published online: 02 November 2021

\section{References}

1. Tiliakos N, DeSorbo J, Martin N, Viti V, Laurence S, Rabin O (2020) A roadmap for obtaining and implementing heat flux measurements in the hypersonic environment. AlAA Paper 2020-2448. https://doi.org/10.2514/6.2020-2448

2. Roy CJ, Blottner FG (2006) Review and assessment of turbulence models for hypersonic flows. Prog Aerosp Sci 42(7-8): 469-530. https://doi.org/10.1016/j.paerosci.2006.12.002

3. Hopkins EJ, Inouye M (1971) An evaluation of theories for predicting turbulent skin friction and heat transfer on flat plates at supersonic and hypersonic Mach numbers. AIAA J 9(6):993-1003. https://doi.org/10.2514/3.6323

4. Huang PG, Coleman GN, Bradshaw P (1995) Compressible turbulent channel flows: DNS results and modelling. J Fluid Mech 305:185-218. https://doi.org/10.1017/S0022112095004599

5. Chen T, Liu T, Wang L, Chen S (2019) Relations between skin friction and other surface quantities in viscous flows. Phys Fluids 31(10):107101. https://doi.org/10.1063/1.5120454

6. Abe H, Antonia RA (2017) Relationship between the heat transfer law and the scalar dissipation function in a turbulent channel flow. J Fluid Mech 830:300-325. https://doi.org/10.1017/jfm.2017.564

7. Kim S, Kim M, Kim K (2002) A direct integration approach for the estimation of time-dependent boundary heat flux. KSME Int J 16(10):1320-1326. https://doi.org/10.1007/BF02983839

8. Ebadi A, Mehdi F, White CM (2015) An exact integral method to evaluate wall heat flux in spatially developing two-dimensional wall-bounded flows. Int J Heat Mass Transf 84:856-861. https://doi.org/10.1016/j.ijheatmasstra nsfer.2014.12.068

9. Fukagata K, Iwamoto K, Kasagi N (2005) Novel turbulence control strategy for simultaneously achieving friction drag reduction and heat transfer augmentation. Proc 4th Int Symp Turbulence and Shear Flow Phenomena. Williamsburg, Virginia, pp 307-312

10. Liu J, Zhao P, Lei M, Yang S, Nemati H (2020) Numerical investigation of spatial-developing turbulent heat transfer in forced convections at different supercritical pressures. Int J Heat Mass Transf 159:120128. https://doi.org/10.1016/j.ijhea tmasstransfer.2020.120128

11. Ghosh S, Foysi H, Friedrich R (2010) Compressible turbulent channel and pipe flow: similarities and differences. J Fluid Mech 648:155-181. https://doi.org/10.1017/S0022112009993004

12. Zhang P, Xia Z (2020) Contribution of viscous stress work to wall heat flux in compressible turbulent channel flows. Phys Rev E 102(4):043107. https://doi.org/10.1103/PhysRevE.102.043107

13. Fukagata K, Iwamoto K, Kasagi N (2002) Contribution of Reynolds stress distribution to the skin friction in wall-bounded flows. Phys Fluids 14(11):L73-L76. https://doi.org/10.1063/1.1516779

14. Renard N, Deck S (2016) A theoretical decomposition of mean skin friction generation into physical phenomena across the boundary layer. J Fluid Mech 790:339-367. https://doi.org/10.1017/ffm.2016.12

15. Sun D, Guo Q, Li C, Liu P (2020) Assessment of optimized symmetric fourth-order weighted essentially non-oscillatory scheme in direct numerical simulation of compressible turbulence. Comput Fluids 197:104383. https://doi.org/10.1016/j. compfluid.2019.104383 
16. Li C, Guo Q, Sun D, Zhang H (2020) Aerothermal prediction of hypersonic flow around spherical capsule model using IDDES approach. Int J Modern Physics B 34(14-16):2040078. https://doi.org/10.1142/S0217979220400780

17. Sun D, Chen J, Li C, Liu P, Guo Q, Yuan X (2020) On the wake structure of a micro-ramp vortex generator in hypersonic flow. Phys Fluids 32(12):126111. https://doi.org/10.1063/5.0030975

18. Sun D, Guo Q, Li C, Liu P (2019) Direct numerical simulation of effects of a micro-ramp on a hypersonic shock wave/ boundary layer interaction. Phys Fluids 31(12):126101. https://doi.org/10.1063/1.5123453

19. Guo Q, Sun D, Li C, Liu P, Zhang H (2020) A new discontinuity indicator for hybrid WENO schemes. J Sci Comput 83:28. https://doi.org/10.1007/s10915-020-01217-w

20. Jiang G, Shu C (1996) Efficient implementation of weighted ENO schemes. J Comput Phys 126(1):202-228. https://doi. org/10.1006/jcph.1996.0130

21. Pirozzoli S, Grasso F, Gatski TB (2004) Direct numerical simulation and analysis of a spatially evolving supersonic turbulent boundary layer at $M=2.25$. Phys Fluids 16(3):530-545. https://doi.org/10.1063/1.1637604

22. Wilcox DC (2006) Turbulence modeling for CFD, 3rd edn. DCW Industries Inc, La Canada

23. Li W, Fan Y, Modesti D, Cheng C (2019) Decomposition of the mean skin-friction drag in compressible turbulent channel flows. J Fluid Mech 875:101-123. https://doi.org/10.1017/ffm.2019.499

24. Hunt J, Wray A, Moin P (1988) Eddies, streams, and convergence zones in turbulent flows. Proceedings of the Summer Program 1988 of the Center for Turbulence Research, N89-24555, pp 193-208

25. Priebe S, Martín MP (2011) Direct numerical simulation of a hypersonic turbulent boundary layer on a large domain. AIAA Paper 2011-3432

26. Duan L, Beekman I, Martín P (2010) Direct numerical simulation of hypersonic turbulent boundary layers. Part 2. Effect of wall temperature. J Fluid Mech 655:419-445. https://doi.org/10.1017/S0022112010000959

27. Pirozzoli S, Bernardini M, Grasso F (2008) Characterization of coherent vortical structures in a supersonic turbulent boundary layer. J Fluid Mech 613:205-231. https://doi.org/10.1017/\$0022112008003005

28. Subbareddy PK, Candler GV (2011) DNS of transition to turbulence in a hypersonic boundary layer. AIAA Paper 20113564. https://doi.org/10.2514/6.2011-3564

\section{Publisher's Note}

Springer Nature remains neutral with regard to jurisdictional claims in published maps and institutional affiliations.

\section{Submit your manuscript to a SpringerOpen ${ }^{\circ}$ journal and benefit from:}

- Convenient online submission

- Rigorous peer review

- Open access: articles freely available online

- High visibility within the field

- Retaining the copyright to your article

Submit your next manuscript at $\boldsymbol{\nabla}$ springeropen.com 